\title{
Dolor Pélvico
}

\author{
Dr. Néstor H. Amorocho \\ Dr. Farid Amastha $\mathrm{H}$.
}

Sic. Ma. del Socorro de Ruíz

Los autores desean expresar su agradecimiento a las entidades, instituciones y personas, que con su colaboración hicieron posible la realización del presente trabajo:

Sociedad Vallecaucana de Obstetricia y Ginecología - Instituto Seguros Sociales - Caja Seccional del Valle - Profamilia - Cali - Hospital de San Juan de Dios - Cali.

Carmen E. Viveros de Salazar - Auxiliar enfermería motivadora y orientadora de las pacientes en cada fase del programa.

\section{Introducción}

Mucho se ha escrito acerca del valor biológico evidente que tiene el dolor para protegernos del daño corporal, llegando a creerse que el dolor está acompañado siempre de una lesión y que es directamente proporcional al grado de ésta (9).

Sabemos que el dolor es sentido de diversas formas, según los estímulos que lo provoquen; también, que en ocasiones se siente en un lugar muy distante de la zona que ha sido lesionada (dolor reflejo).

Aunque en años recientes los ginecólogos se han dado cuenta, cada vez más, que los trastornos emocionales pueden alterar la función y producir dolor en la región pélvica, son muchas las mujeres que para curarse de molestias ginecológicas se someten a operaciones como suspensión del útero, legrados, amputación cervical, operaciones plásticas, apendicectomías e incluso histerectomías. Desgraciadamente, el tratamiento de los trastornos ginecológicos psicógenos y de las alteraciones en el funcionamiento sexual, por medio de procedimientos operatorios o de otro tipo físico, no es inocuo sino que fija y refuerza la idea de enfermedad (16).

El dolor pélvico es una de las más comunes quejas entre las mujeres jóvenes y de mediana edad que asisten a la consulta externa. Es un síntoma difícil de tratar porque a menudo la causa orgánica del dolor no puede encontrarse.

El dolor pélvico crónico con examen ginecológico negativo (palpación bimanual) puede resultar inexplicable para el médico y muy molesto para la paciente.

Comúnmente el médico diagnostica enfermedad inflamatoria pélvica teniendo por resultado que la mujer recibe series de tratamientos antibióticos y no obstante el dolor recurre. Como alternativa, después que prolongada investigación no ha revelado patología, se efectúa operación la cual permite aclarar la situación pélvica (2). 
El recurso prematuro de la cirugía es el error característico en el tratamiento de pacientes con dolor pélvico (19).

Entre las mujeres que consultan por dolor ginecológico crónico hay significativo porcentaje en quienes la queja no tiene clara base orgánica. Este problema es bien conocido en la práctica ginecológica. En la literatura se le ha llamado de muy diversas maneras, y Naujoks hace años, estuvo hablando de la enfermedad con 20 diferentes nombres. Bajo estas circunstancias parece lógico pretender aclarar si los factores psicogenéticos contribuyen al origen o al mantenimiento de esta condición (18).

Hipócrates explicaba el dolor, al imaginar que la matriz se desplazaba, lo cual causaba presión de vasos sanguíneos y otros órganos (3).

Galeno decía que la histeria resultaba de congestión uterina por el líquido menstrual o por sangre.

Noyes expresa en su tratado de psiquiatría general: "No es sorprendente que la neurosis y los síntomas psicosomáticos se presenten con frecuencia en un sistema de importancia biológica, tan fundamental, relacionado con instinto, tan elemental como el genitourinario, durante el desarrollo de la personalidad". Las ideas y las emociones que se relacionan con el funcionamiento del aparato genitourinario se van cargando cada vez más de sentimientos de vergüenza, culpa, miedo, asco e incluso odio, todo como resultado de una educación defectuosa (16).

Breuer anotaba en 1882: "Así creo los dolores ováricos dependen del aparato genital, el que sean mediados síquicamente habría que demostrarlo y no es suficiente que este dolor pueda ser provocado como alucinación en la hipnosis o que los dolores ováricos puedan ser también de origen síquico. Se producen como el eritema, o como una de las secreciones normales, tanto por causas síquicas como puramente so- máticas"; y añade posteriormente: "en las histéricas femeninas el dolor de ovarios conduce al diagnóstico de peritonitis" (5)

Sin duda alguna muchas pacientes neuróticas localizan molestias en la pelvis, cuando no existe ninguna lesión orgánica. De otra parte, muchas han sido calificadas como neuróticas porque el ginecólogo no tuvo suficiente habilidad para detectar un trastorno poco manifiesto. En 17 casos estudiados por culdoscopia, cuyo origen del dolor no se podía explicar por palpación bimanual, se logró en unos visualizar la causa del dolor y corregirlo, en tanto que en otros pudo establecerse que las vísceras eran normales.

A veces, la seguridad de saber que no existía enfermedad orgánica bastó para dar a la paciente tranquilidad suficiente. Cuando se hizo necesario recurrir a tratamiento siquiátrico, el conocimiento de la falta absoluta de patología pelviana, ayudó a la labor del psiquiatra $(20)$.

Diez años antes de Deutch (1922) sólo se hablaba de neurosis de óryanos y psicosis de órganos (14).

Hoy en día las relaciones psicosomáticas se reconocen como realidad clínica, sobre todo en medicina interna, pediatría o ginecología (14) .

Tradicionalmente se han designado los trastornos psicosomáticos como histeria de conversión o reacción de conversión, y es frecuente que los autores de trabajos e investigaciones, relacionadas con el Dolor Pélvico Psicógeno, empleen estos términos que se definen como la conversión de la angustia en síntomas funcionales que afectan los órganos o partes del cuerpo, inervados por el sistema nervioso sensoriomotor, sirviendo para prevenir o disminuir cualquier angustia consciente o palpable y suelen simbolizar el conflicto mental primario que produjo la angustia. Esto proporciona además una ventaja secundaria (16) 
Históricamente el término psicosomático fue utilizado por primera vez en 1818 por el médico alemán Heinroth, aunque ya la imposibilidad de disociar el alma de la salud del cuerpo se vislumbra en el libro de Job y en la obra de Platón (14)

En un grupo numeroso de pacientes con dolor pélvico, sin patología obvia se mostró que cuando esas mujeres eran ansiosas había un aumento en la cantidad de sangre menstrual. Se propuso que esa "congestión vascular crónica", es un componente psicosomático a menudo coexistente con inmadurez e inseguridad emocional ( 7 ).

La crítica mayor para este estudio fue que como no se disponía de laparoscopio era imposible asegurar que no había patología pélvica en estas pacientes.

Con el advenimiento y la disponibilidad de laparoscopio ha sido posible distinguir más claramente entre las causas orgánicas y psicosomáticas de dolor pélvico (2).

Benson (3) en 1965 establece que el dolor pélvico persistente y atípico en cuanto a la mayor parte de las entidades ginecológicas reconocidas, es sicógeno. Para Benson (18) el dolor "funcional" invariablemente es signo de problemas emocionales de larga data que exigen solución. En nuestro medio, en el cual se destaca la importancia del afecto, el dolor pélvico sin causa determinante corresponde a personalidad histérica, la cual es casi exclusiva de la mujer.

Mandy (15) afirma: "Muchas mujeres que buscan ayuda para problemas de la mujer son en realidad, mujeres con problemas". Los problemas de la mujer no son ginecológicos ni siquiátricos sino ambas cosas.

Las molestias dolorosas a menudo son de naturaleza histérica y en oca- siones pueden ser de tipo alucinatorio; al igual que los otros síntomas histéricos, el paciente relata un cuadro complejo o poco habitual y los datos no concuerdan con los que suelen derivarse de una enfermedad física (16).

La dismenorrea habitual suele ser la causa de conflictos internos profundos en la mayoría de los casos; afecta a mujeres neuróticas, frígidas y de difícil adaptación, que temen conscientemente al acto sexual. Puede tratarse de personalidades viriles, dominadoras que se sienten rebajadas cuando les Ilega la menstruación, otras se han quedado en su comportamiento afectivo en el estado pasivo del lactante, buscan protección materna y se asustan ante los deberes que como mujeres deben afrontar (14).

El dolor es necesidad anormal de solicitud o satisfacción en la mujer masoquista, resentimiento en la frustrada, ansiedad en la hipocondríaca, culpa en la exigente o introspectiva, o mecanismo típico de defensa en la que linda con la esquizofrenia (18).

En las pacientes que consultan por dolor pélvico crónico en el $25 \%$ de los casos aproximadamente, no se descubre anomalía física; en el resto se encuentran lesiones mínimas o variación física insignificante pero no relacionada con la severidad del dolor (3).

En los últimos 50 años se han descubierto alteraciones en lo anatomofisiológico de las vísceras pélvicas, en aquellas pacientes de dolor pélvico inexplicado. A pesar de muchos ensayos, el dolor no puede estimarse objetivamente ya que es un fenómeno emocional y subjetivo (3).

A pesar de que la endoscopia pélvica (laparoscopia y culdoscopia), se establecieron en la práctica médica por Kalk (13) en 1925 y Telinde (20) en 1936, y la metodología en el diagnósti- 
co ginecológico, gracias los subsiguientes avances para mejorar la calidad de los instrumentos, brindó a la ginecología el aumento de medios de diagnóstico, no se encontraba en la literatura hasta años muy recientes, década de los setenta, una utilización de ellos para aclarar el problema del dolor pélvico, atribuido, casi exclusivamente, a factores sicoemocionales en épocas anteriores.

Al tiempo que nosotros, nos interesábamos en el problema del dolor pélvico crónico sin diagnóstico definido, Beard y Colab (2) publican su trabajo titulado "Dolor pélvico crónico en la mujer", siguiendo un esquema muy similar al propuesto por nosotros en diciembre de 1977.

Motivados por las consideraciones anteriores, se propuso realizar una evaluación racional, del dolor pélvico, contemplando los aspectos clínicos y sicológicos de un grupo de pacientes, en forma prospectiva, utilizando la laparoscopia como medio objetivo para determinar patología pélvica.

\section{MATERIAL Y METODOS}

\section{Definición}

Para efectos relacionados con este estudio, definimos dolor pélvico así: "Síntoma manifestado por la mujer, relacionado o no con el ciclo menstrual, de 3 o más meses de evolución, motivo de consulta ginecológica al menos una vez, sin que se haya establecido un diagnóstico etiológico que lo justifique".

\section{Universo de trabajo}

Se propuso inicialmente el estudio de 100 pacientes, que reunieran las condiciones de la definición anterior. Estas pacientes provendrian de la remisión efectuada por los miembros de la Sociedad Vallecaucana de Obstetricia y Ginecologia, o entidades en las cuales ellos laboran: ISS, HUV. HSJD. Profamilia.

El universo de trabajo se redujo a 42 pacientes, estudiadas en el lapso comprendido entre el 15 de abril y el 15 de septiembre de 1979, debido a que gran número de las pacientes remitidas, no reunian las condiciones de la definición.

\section{Recursos físicos y humanos}

En la sede de Profamilia en Cali, se centralizó el grupo de trabajo, y las pacientes allí remitidas, gracias a las facilidades locativas y de equipo de la Institución, fueron conducidas en el desarrollo del programa.

El personal de investigación fue conformado por dos médicos ginecólogoslaparoscopistas, una sicóloga y una auxiliar de enfermería, con funciones de orientación, dar citas y programar las pacientes.

\section{Mecánica}

Se diseñó un formato de remisión que contempla aspectos de identificación, socio-demográficos, antecedentes médico y gineco-obstétricos, características clínicas del dolor, y examen ginecológico. Todas las pacientes fueron evaluadas en los diferentes aspectos mencionados. 


\section{CORRELATO OFICIAL SOCIEDAD VALLECAUCANA \\ OBSTETRICIA Y GINECOLOGIA}

\section{XIII - CONGRESO NACIONAL DE OBSTETRICIA Y GINECOLOGIA DOLOR PELVICO}

\section{A. - NOMBRE:}

Dirección residencial:

1. - Edad:

2.-- Raza:

3. - Estado civil:

4.- Nivel educacional:

\section{B. - MENARCA:}

1. - Ciclos menstruales:

2. - Paridad:

3.- Cirugía previa:

4.- Tratamiento médico previo:

\section{C. - DOLOR:}

1.- Tiempo de aparición:

2.- Relación con el ciclo:

3.- Localización:

Meses:

4.- Características:

Cólico:

Agudo:

5.- Irradiación:

Incapacitante:

Otros:

\section{D. - EXAMEN GINECOLOGICO:}

1.- Celes:

2.- Cerviz:

3. - Cuerpo uterino:

4. - Posición uterina:

5. - Fondos de saco:

6.- Anexos:

\section{E. - EVALUACION EMOCIONAL:}

Favor remitir la paciente con este protocolo:

A: Profamilia - Cali

Dirección: Calle $23 \mathrm{~N}$ No. $3 \mathrm{~N}-40$

Teléfonos: $631730-684984$ 
Desde el punto de vista sicológico, fueron sometidas a encuesta clínica hecha por la sicóloga y a la realización de un cuestionario que contempla los siguientes aspectos: socialización, productividad, autoimagen, antecedentes traumáticos, antecedentes familiares, vida afectiva, ventajas del dolor y actitud de la paciente.

Del total de pacientes, a 31 (11 no se presentaron) se aplicó el test M.M. P.I. (Minnesota Multiphasic Personality Inventory) (12); instrumento sicométrico, diseñado para proveer en una sola prueba, puntajes de las fases más importantes de la personalidad, distribuidas en escalas.

\section{A - Cuatro para la validez:}

? =- Puntaje de duda

$\mathrm{F}=$ Puntaje de validez

$\mathrm{L} \quad=$ Escala de mentira

$\mathrm{K}=$ Puntaje de corrección

\section{B.- Diez escalas clínicas:}

$\mathrm{Hs}=$ Hipocondriasis
$\mathrm{Hy}=$ Histeria
$\mathrm{Mf}=$ Masculinidad femeneidad
$\mathrm{Pt}=$ Sicastenia
$\mathrm{Ma}=$ Hipomanía
$\mathrm{D}=$ Depresión
$\mathrm{Pd}=$ Desviación sicopática
$\mathrm{Pa}=$ Paranoia

$$
\begin{aligned}
& \mathrm{Sc}=\text { Esquizofrenia } \\
& \mathrm{S}=\text { Socialización }
\end{aligned}
$$

Al finalizar esta fase la sicóloga realizó una evaluación de cada paciente. para llegar a establecer un diagnóstico sicológico.

Las pacientes fueron luego a laparoscopia diagnóstica, realizada por los investigadores.

Estas laparoscopias fueron calificadas como positivas y negativas, según se observara patología relacionada con el síntoma dolor, o no. Se llenó el formato que para el efecto tiene diseñado la Sociedad Colombiana de Médicos Endoscopistas .

Todas las pacientes fueron revaluadas médicamente, en control posterior, para informarles del resultado de su estudio, orientarlas sobre la conducta a seguir; se enviaron al médico remitente los resultados del estudio efectuado.

\section{RESULTADOS}

Siguiendo la metodología propuesta en la cual la laparoscopia es el medio objetivo del problema, tanto los aspectos médico-clínicos, como los sicológicos, fueron cruzados con los resultados laparoscópicos, de acuerdo con el diagnóstico como positiva o negativa.

(Ver formatos en las páginas siguientes) 
Name Age

Sex

Date Tested

Addreas Occupation

Education

Marital Status.

Relerred by
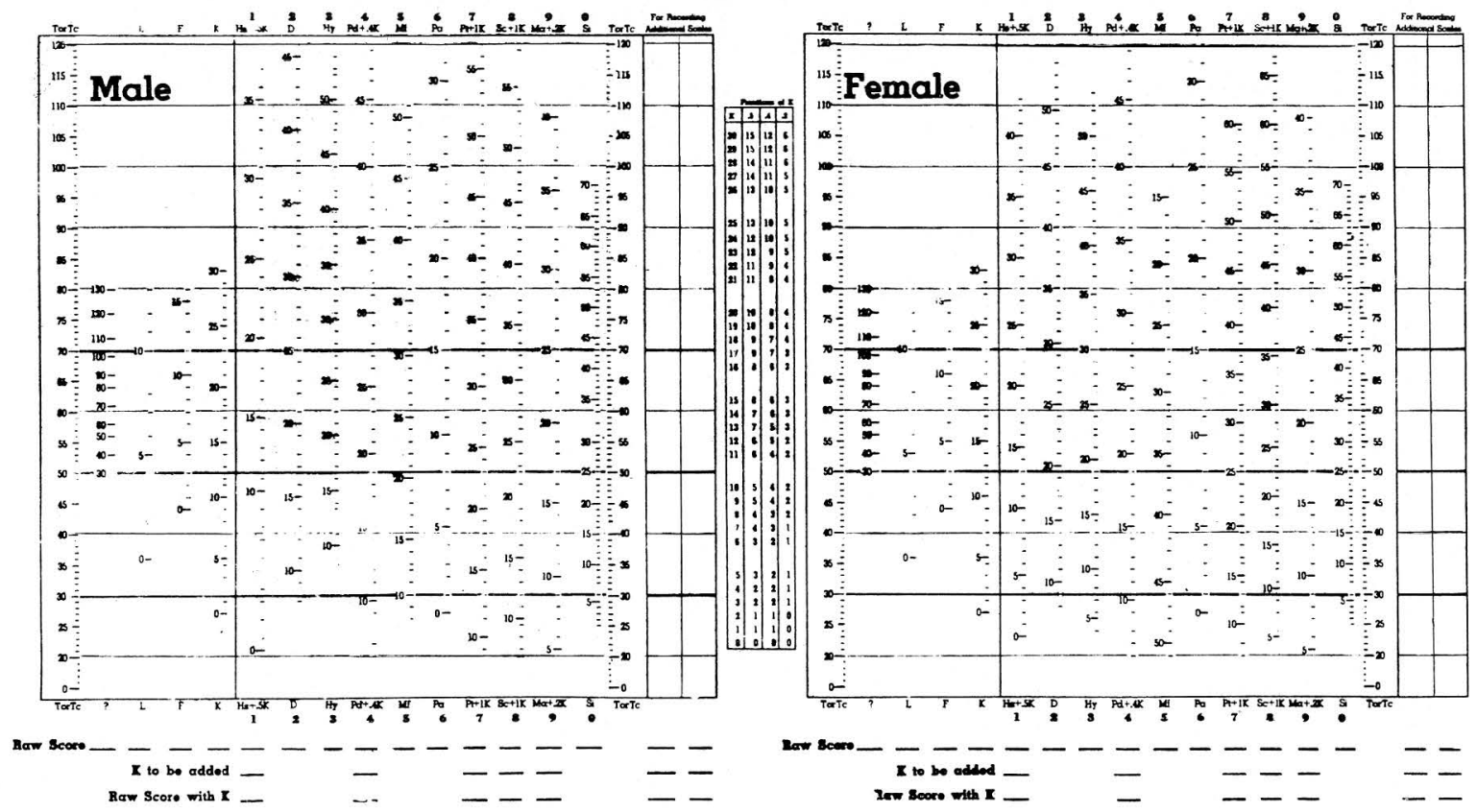
and

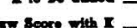
$--$
$-$

Raw Scoro with X

Date 


\section{SOCIEDAD COLOMBIANA MEDICOS ENDOSCOPISTAS}

C A L I

\section{INFORME DE LAPAROSCOPIA DIAGNOSTICA}

Paciente:

Laparoscopista Dr

Anestesiólogo Dr.

Clínica:

Anestesia:

Fecha:

\section{E S C R I P C I O N}

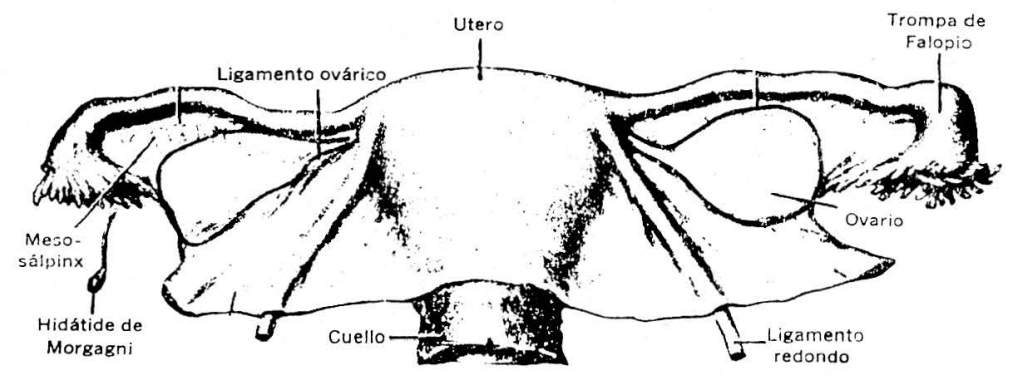

Peritoneo:

Ligamentos:

Utero:

Ovario derecho:

Trompa derecha:

Ovario izquierdo:

Trompa izquierda:

Permeabilidad tubaria

Otros:

Diagnóstico final:

Recomendaciones:
Redondo

Utero sacro

Der.

Izq.

\section{CLASIFICACION SOCIO-DEMOGRAFICA}

\section{CUADRO 1}

\section{A. - Edad}

En él encontramos que el dolor pélvico se presenta en nuestras pacientes en la etapa reproductiva; en cuanto al diagnóstico laparoscópico se observa que el número porcentual de laparos. copias negativas aumenta en relación con la edad, en tanto que en aquellas con diagnóstico positivo, disminuye con la edad.

\section{B.- Clase socio-económica}

Se tomó como base de esta clasificación el trabajo realizado por Bernal (4) publicado en 1969 el cual mostró la distribución por ingresos de la pobla- 
CUADRO 1

\section{DOLOR PEL VICO (42 PTES.) CLASIFICACION SOCIO-DEMOGRAFICA}

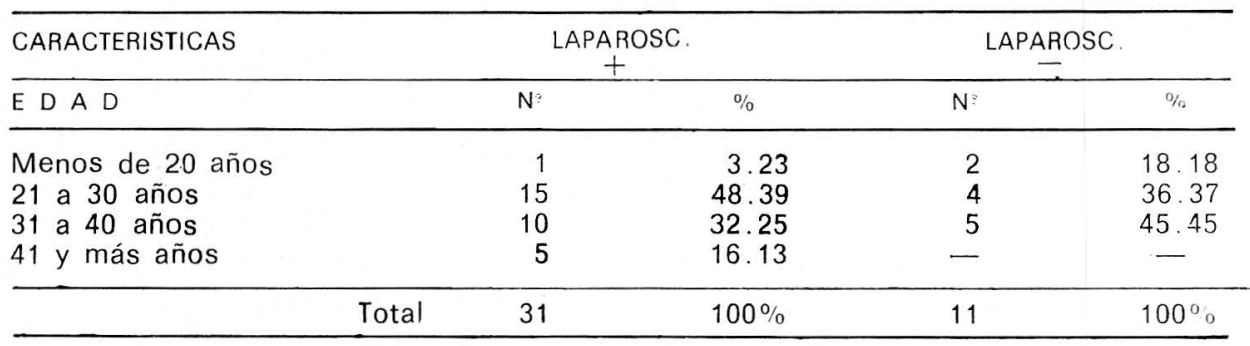

NOTA: Relación directa en negativas. Relación inversa en positivas.

\begin{tabular}{lrrrr}
\hline CLASE SOCIO-ECONOMICA & $\mathrm{N}$ & $\%$ & $\mathrm{~N}$ & $\%$ \\
\hline A: Alta & - & & - & - \\
B: Media alta & 4 & 12.90 & 1 & 9.09 \\
C: Media & 8 & 25.81 & 5 & 36.37 \\
D: Baja & 15 & 48.39 & 1 & 45.45 \\
Fuera de la ciudad & 3 & 9.67 & - & 9.09 \\
Sub-registro & 1 & 3.23 & 11 & $100 \%$ \\
\hline
\end{tabular}

NOTA: Diferencia significante en clase media.

\begin{tabular}{lrccc}
\hline PARIDAD & $\mathrm{N}$ & $\%$ & $\mathrm{~N}$ & $\%$ \\
\hline Nulíparas & 12 & 38.71 & 3 & 27.27 \\
Primíparas & 5 & 16.13 & 2 & 18.18 \\
Multíparas & 14 & 45.16 & 6 & 54.55 \\
\hline & & $100 \%$ & 11 & $100 \%$ \\
\hline
\end{tabular}

NOTA: La mayor incidencia de multiparas es negativa.

ción de Cali, proyectándolo a 10 anos después.

En relación con el diagnóstico laparoscópico podemos apreciar una mayor incidencia de laparoscopias negativas en la clase media $36.37 \%$ frente a $25.81 \%$ en las positivas.

\section{C.- Paridad}

La mayor incidencia de laparoscopias negativas se presenta en el grupo de multíparas; nótese que edad y paridad tienen igual significancia en nuestro grupo de estudio. 
CUADRO 2

\section{DOLOR PELVICO (42 PTES.) CLASIFICACION SOCIO-DEMOGRAFICA}

\begin{tabular}{lrrrr}
\hline CARACTERISTICAS & \multicolumn{2}{c}{ LAPAROSC } & \multicolumn{2}{c}{ LAPAROSC. } \\
\hline EDUCACION & $\mathrm{N}$ & \multicolumn{1}{c}{$\%$} & $\mathrm{~N}$ & $\%$ \\
\hline Analfabetas & 1 & 3.23 & - & - \\
Primaria & 13 & 41.94 & 7 & 63.64 \\
Bachillerato & 14 & 45.16 & 4 & 36.36 \\
Universidad & 3 & 9.67 & - & - \\
\hline & & $100 \%$ & 11 & $100 \%$ \\
\hline
\end{tabular}

NOTA: Menor nivel muestra mayor incidencia en laparoscopias negativas $63.64 \%$.

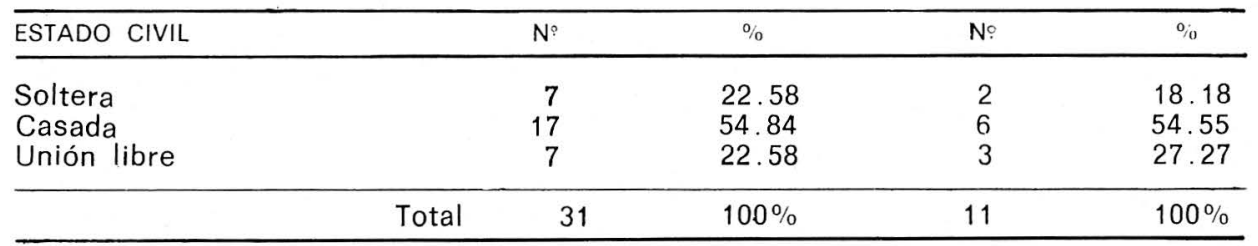

NOTA: No muestra diferencia significante en los grupos.

\begin{tabular}{|c|c|c|c|c|c|}
\hline$R A Z A$ & & $\mathrm{~N}^{\circ}$ & $\%$ & No & $\%$ \\
\hline $\begin{array}{l}\text { Blanca } \\
\text { Mestiza } \\
\text { Negra }\end{array}$ & & $\begin{array}{r}16 \\
13 \\
2\end{array}$ & $\begin{array}{r}51.61 \\
41.94 \\
6.45\end{array}$ & $\begin{array}{l}5 \\
5 \\
1\end{array}$ & $\begin{array}{r}45.45 \\
45.45 \\
9.10\end{array}$ \\
\hline & Total & 31 & $100 \%$ & 11 & $100 \%$ \\
\hline
\end{tabular}

NOTA: No difieren significantemente

\section{A.- Educación}

Se encuentra mayor incidencia de negativas en las pacientes de bajo nivel educacional.

Creemos que a medida que aumenta el nivel educacional existe una mayor capacidad para identificar el dolor orgánico .

\section{B.- Estado civil}

Encontramos un porcentaje predominante en las casadas, en ambos grupos, sin que exista diferencia porcentual.

C.- Raza

Nuestro estudio agrupó pacientes de raza mestiza y blanca existiendo un ligero predominio de la última en las laparoscopias positivas. En las laparoscopias negativas no hay diferencia. 
CUADRO 3

DOLOR PELVICO (42 PTES.)

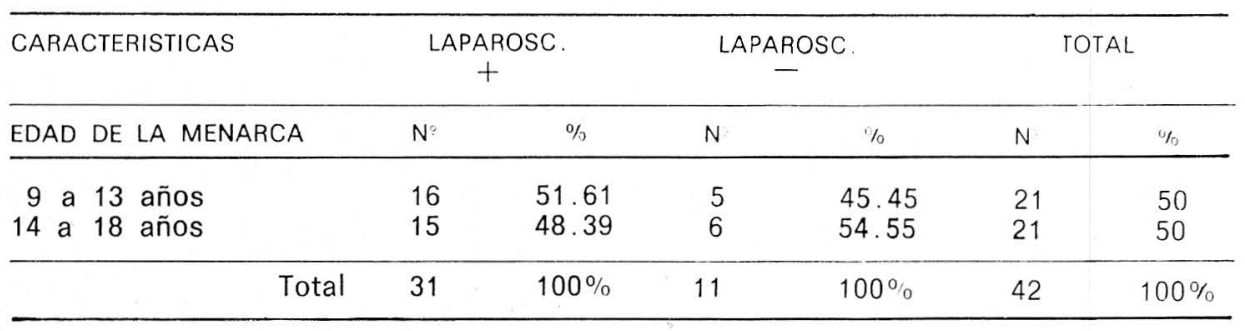

NOTA: No hay diferencia fundamental en los grupos.

\begin{tabular}{|c|c|c|c|c|c|c|}
\hline CICLO MENSTRUAL & № & $\%$ & $N^{2}$ & $\%$ & $N^{\prime}$ & $\%$ \\
\hline Normal & 19 & 61.29 & 8 & 72.73 & 27 & 64.28 \\
\hline Oligomenorrea & 7 & 22.58 & 1 & 9.09 & 8 & 19.05 \\
\hline Poli-hipermenorrea & 5 & 16.13 & 2 & 13.18 & 7 & 16.67 \\
\hline Total & 31 & $100 \%$ & 11 & $100 \%$ & 42 & $100 \%$ \\
\hline
\end{tabular}

NOTA: Incidencia normal significativa en grupo negativo.

\begin{tabular}{|c|c|c|c|c|c|c|}
\hline APARICION DEL DOLOR & $\mathrm{N}$ & $\%$ & $\mathrm{~N}$ & $\%$ & $\mathrm{~N}$ & $\%$ \\
\hline \multirow{2}{*}{$\begin{array}{l}\text { Relacionado con el ciclo } \\
\text { No relacionado con el } \\
\text { ciclo }\end{array}$} & 13 & 41.94 & 7 & 63.64 & 20 & 47.62 \\
\hline & 18 & 58.06 & 4 & 36.36 & 22 & 52.38 \\
\hline Total & 31 & $100 \%$ & 11 & $100 \%$ & 42 & $100 \%$ \\
\hline
\end{tabular}

NOTA: Grupo negativo relacionado con ciclo 63.64 es significante.

\section{A.- Edad de la menarca}

No encontramos diferencia significante entre ambos grupos con respecto a la edad de la menarca.

\section{B.- Ciclo menstrual}

Se destaca la alta incidencia de pacientes con ciclo menstrual normal, en el grupo laparoscopias negativas $(72.73 \%)$.

\section{C.- Aparición del dolor}

Llama la atención la relación del dolor con el ciclo menstrual, $63.64 \%$ encontrado en el grupo de laparoscopias negativas. A pesar que en los totales la distribución de relación del dolor o no, con el ciclo menstrual, son muy cercanas. 


\section{CUADRO 4 \\ DOLOR PELVICO (42 PTES.) \\ CARACTERISTICAS}

\begin{tabular}{|c|c|c|c|c|c|}
\hline \multirow{2}{*}{$\begin{array}{l}\text { CARACTERISTICAS } \\
\text { LOCALIZACION }\end{array}$} & \multicolumn{3}{|c|}{$\begin{array}{c}\text { LAPAROSC. } \\
+\end{array}$} & \multicolumn{2}{|c|}{ LAPAROSC. } \\
\hline & & $\mathrm{N}$ & $\%$ & $\mathrm{~N}^{\circ}$ & $\%$ \\
\hline $\begin{array}{l}\text { Hipogastrio } \\
\text { Fosas iliacas } \\
\text { Difuso }\end{array}$ & & $\begin{array}{r}12 \\
16 \\
3\end{array}$ & $\begin{array}{r}38.71 \\
51.61 \\
9.68\end{array}$ & $\begin{array}{l}3 \\
7 \\
1\end{array}$ & $\begin{array}{r}27.27 \\
63.64 \\
9.09\end{array}$ \\
\hline & Total & 31 & $100 \%$ & 11 & $100 \%$ \\
\hline
\end{tabular}

NOTA: Sensiblemente igual en ambos'grupos.

\begin{tabular}{lcccc}
\hline TIPO DE DOLOR & $\mathrm{N}$ & $\%$ & $\mathrm{~N}$ & $\%$ \\
\hline Cólico & 9 & 29.03 & - & - \\
Agudo & 6 & 19.36 & 8 & 72.73 \\
Incapacitante & 5 & 16.13 & - & - \\
Sordo & 7 & 22.58 & 2 & 18.18 \\
Otros & 4 & 12.90 & 1 & 9.09 \\
\hline & 31 & $100 \%$ & 11 & $100 \%$ \\
\hline
\end{tabular}

NOTA: Predominancia de agudo en el grupo negativo $72.73 \%$.

\begin{tabular}{lrrrr}
\hline IRRADIACION & $\mathrm{N}$ & $\%$ & $\mathrm{~N}$ & \multicolumn{1}{c}{$\%$} \\
\hline Negativa & 8 & 25.81 & 3 & 27.27 \\
Espalda & 18 & 58.06 & 6 & 54.55 \\
Miembros inferiores & 2 & 6.45 & 1 & 9.09 \\
Abdomen & 2 & 6.45 & 1 & 9.09 \\
Otros & 1 & 3.23 & - & - \\
\hline & & $100 \%$ & 11 & $100 \%$ \\
\hline
\end{tabular}

NOTA: No hay diferencia significante en los grupos.

\section{A.- Localización}

En el grupo de laparoscopias negativas se aglutina en "fosas iliacas" $63.64 \%$ y "difuso" sólo alcanzó un $9.09 \%$; nótese que en el grupo de laparoscopias positivas la cifra porcentual en el mismo renglón es más baja.

\section{B.- Tipo de dolor}

Encontramos un muy alto predominio de "agudo" en las laparoscopias nega- tivas $72.73^{\circ}$ y y una amplia distribución de la descripción del dolor de las laparoscopias positivas.

\section{C.- Irradiación}

Se observa un predominio en ambos grupos con irradiación a "espalda", pero comparativamente no encontramos diferencia $(58.06 \%$ y $54.55 \%)$. 


\section{CUADRO 5}

DOLOR PELVICO (42 PTES.)

HALLAZGOS EN LAPAROSCOPIAS +

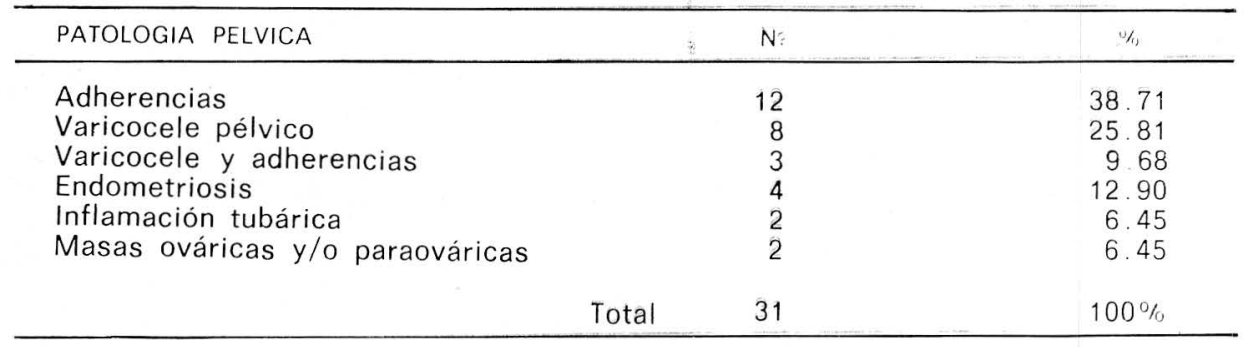

NOTA: Coincidente con DX anteriores 3 pacientes presentaron pequeños miomas uterinos

Adherencias, varicocele pélvico y su combinación sumaron el $74.20 \%$ de los

hallazgos positivos de este estudio

CUADRO 6

DOLOR PELVICO (42 PTES.)

ANTECEDENTES QUIRURGICOS

\begin{tabular}{|c|c|c|c|c|c|}
\hline CIRUGIAS PREVIAS & & $\begin{array}{c}\text { Laparosc. } \\
-\end{array}$ & $\begin{array}{c}\text { Laparosc. } \\
+\end{array}$ & \multicolumn{2}{|c|}{ HALLAZGOS } \\
\hline Esterilización tubárica & & 1 & 3 & $\left\{\begin{array}{l}\text { Adherencias } \\
\text { Varicocele }\end{array}\right.$ & $\begin{array}{l}2 \\
1\end{array}$ \\
\hline Cesárea & & & 1 & $\left\{\begin{array}{l}\text { Adherencias } \\
\text {. }\end{array}\right.$ & 1 \\
\hline $\begin{array}{l}\text { Laparoscopia } \\
\text { Ooforectomía }\end{array}$ & & 1 & 1 & $\begin{array}{l}\text { Endometriosis } \\
\text { Varicocele }\end{array}$ & $\begin{array}{l}1 \\
1\end{array}$ \\
\hline Cuña de ovarios & & 1 & 3 & $\left\{\begin{array}{l}\text { Varicocele }\end{array}\right.$ & $\begin{array}{l}3 \\
1\end{array}$ \\
\hline $\begin{array}{l}\text { Histerectomía } \\
\text { Plastia vaginal } \\
\text { Apendicectomía }\end{array}$ & & $\begin{array}{l}1 \\
2 \\
1\end{array}$ & 1 & Varicocele & 1 \\
\hline & Total & 7 & 9 & & \\
\hline
\end{tabular}

NOTA: Relación directa de antecedentes con laparoscopia positiva.

Llama la atención el hecho de la aparición de adherencias pélvicas, en alto porcentaje, en las pacientes con ante- cedentes de cirugía tan frecuentes, como esterilización tubaria (2 de 4) y cuña de ovarios (3 de 4). 
GRAFICO No. 7

\section{DOLOR PELVICO (42 PTES.) \\ LAPAROSCOPIA POSITIVA (22 PTES.)}

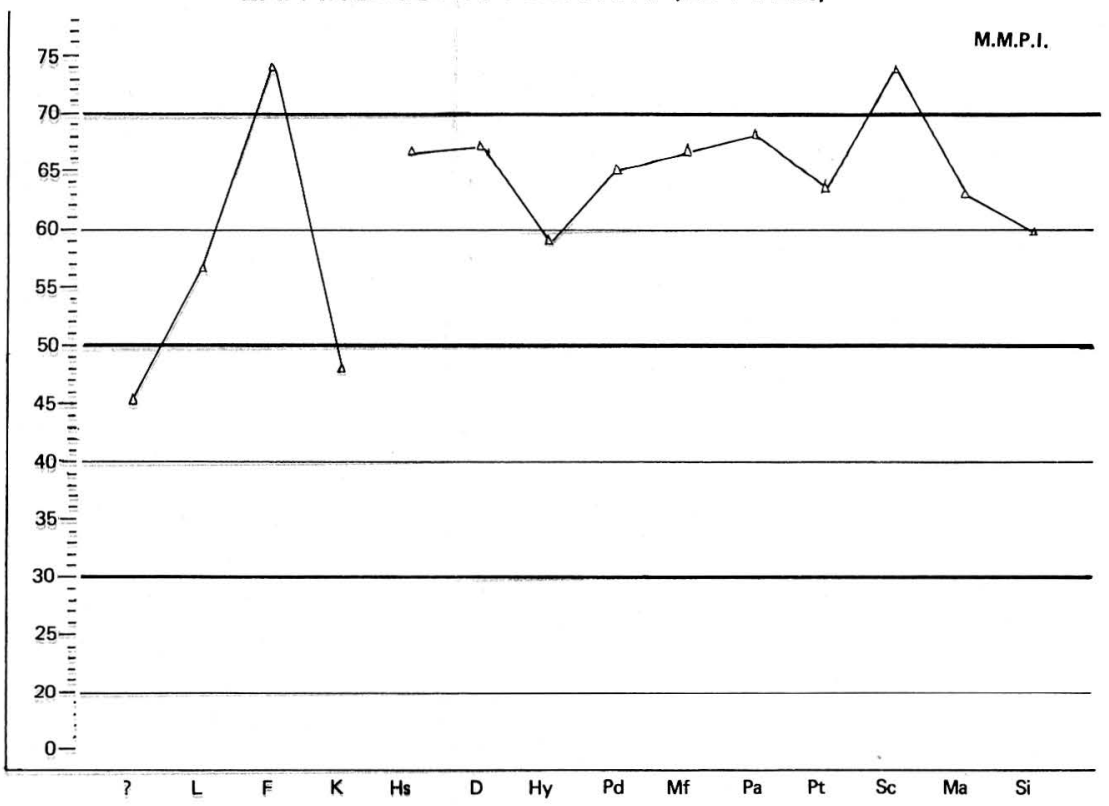

\section{RESULTADOS Y DIAGNOSTICOS MMPI}

En las escalas clínicas la prueba nos señala una tendencia o igualdad de puntaje para los dos grupos, encontrándose sólo una diferencia en algunas escalas de 1 ó 2 puntos.

Se encontraron cuatro escalas donde los puntajes son iguales. HS - PA - MF PD.

Las pacientes del grupo laparoscopia negativa muestran sindrome depresivo, también manifiestan sentimientos de inutilidad, falta de confianza en sí mismas e introversión.

El grupo laparoscopia positiva muestra alta tendencia a la depresión.
Los dos grupos presentan alto puntaje en la escala Sc. (esquizofrenia) indicativo de comportamiento de tipo sicótico.

Considerando las dos escalas de puntuación más alta en los grupos negativos y positivos (D - Es) podremos decir que todas las pacientes presentaron trastornos de tipo afectivo.

La escala de validez nos da un índice fiable de la validez de la prueba; no obstante ser el puntaje $F$ significativamente alto para los dos grupos, esto no nos autoriza para invalidar completamente la prueba, pero si para tomar sus puntajes con reserva. Por otra parte el alto puntaje de $\mathrm{F}$ es indicativo de descuido de los sujetos al contestar la prueba, o para comprensión de los ítems que en ellas se encuentra. 
GRAFICO No. 8

DOLOR PELVICO (42 PTES.)

LAPAROSCOPIA NEGATIVA (9 PTES.)

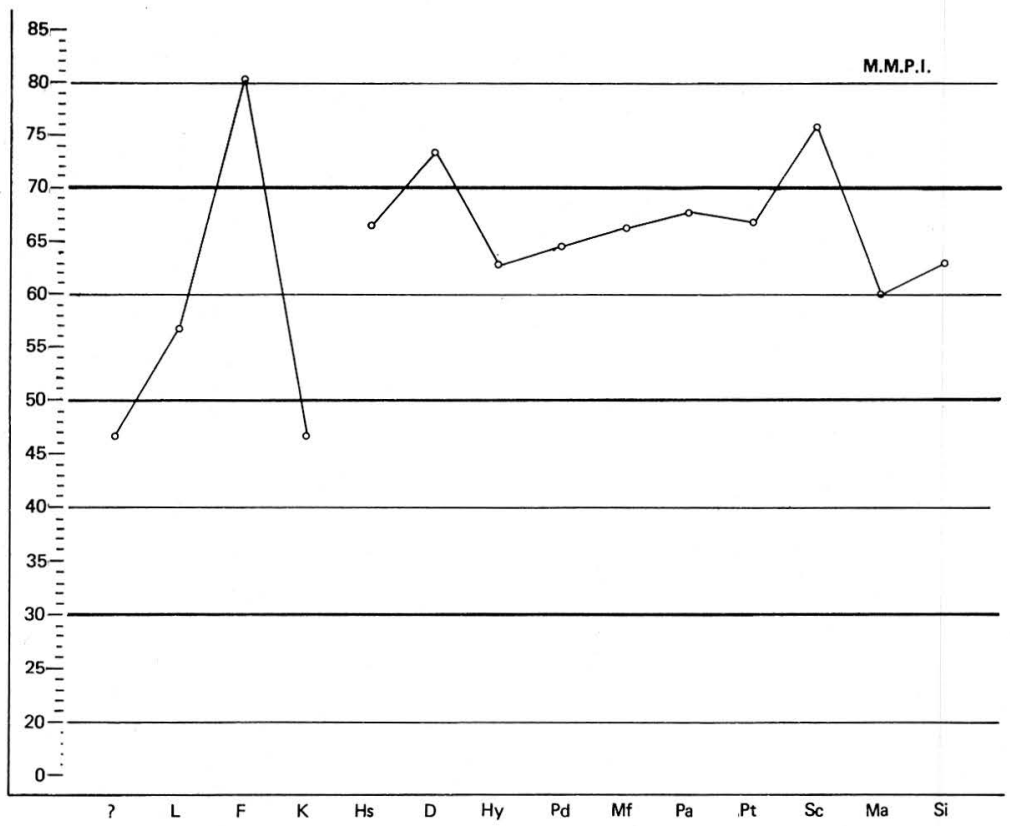

\section{Resultados de entrevistas y aplicación} de cuestionario

Para el análisis de estos resultados se tomó como base la última clasificación hecha por la "APA" (Asociación Psiquiátrica Americana) respecto a trastornos de la personalidad y del área siconeurótica que se definen así:

\section{Trastorno de personalidad}

Se designan los casos en los que la personalidad en lugar de utilizar síntomas que se expresen en alteraciones mentales somáticas o emocionales, en su esfuerzo por lograr adaptarse utilizan patrones de acción o de conducta.

Basándose en la dinámica del desarrollo de la personalidad, se caracteri- zan por defectos en el desarrollo de la personalidad o por tendencia patológica de su estructura. Presentan poca angustia, depresión, inestabilidad emocional, poca responsabilidad para consigo mismo y la sociedad, exageraciones en su estado de ánimo, aislamiento, obsesión, compulsión o reacción agresiva.

\section{Trastornos siconeuróticos}

Comprende un grupo relativamente benigno de trastornos de la personalidad, los cuales pueden describirse como alteraciones intermedias.

Surgen del esfuerzo que el individuo hace para mejorar problemas sicológicos internos privados y específicos y situaciones que provocan "stress", que el paciente mismo es incapaz de dominar 
sin tensión o recursos sicológicos in. quietantes provocados por la angustia que ha estimulado.

Sus sintomas más frecuentes son: angustia o esfuerzos por controlar a tra- vés de las reacciones de conversión fobias $u$ obsesiones y compulsiones: inadaptaciones a través de las etapas de su vida evolutiva, conflictos perma nentes con su medio, emocionales y agresividad.

CUADRO 9

DOLOR PELVICO (42 PTES.)

EVALUACION SICOLOGICA

\begin{tabular}{|c|c|c|c|c|c|}
\hline \multicolumn{2}{|c|}{ A SPECTOS } & \multicolumn{2}{|c|}{$\begin{array}{c}\text { LAPAROSC. } \\
+ \\
\end{array}$} & \multicolumn{2}{|c|}{$\begin{array}{c}\text { LAPAROSC } \\
-\end{array}$} \\
\hline & & $N$ & $\%$ & $\mathrm{~N}^{\cdot}$ & $\%$ \\
\hline Socialización & $\begin{array}{l}\text { Normal } \\
\text { Anormal }\end{array}$ & $\begin{array}{l}17 \\
14 \\
\end{array}$ & $\begin{array}{l}54.84 \\
45.16\end{array}$ & $\begin{array}{l}5 \\
6\end{array}$ & $\begin{array}{l}45.45 \\
54.55\end{array}$ \\
\hline Productividad & $\begin{array}{l}\text { Normal } \\
\text { Anormal }\end{array}$ & $\begin{array}{l}18 \\
13\end{array}$ & $\begin{array}{l}58.06 \\
41.94\end{array}$ & $\begin{array}{l}8 \\
3\end{array}$ & $\begin{array}{l}72.73 \\
27.27 \\
\end{array}$ \\
\hline Autoimagen & $\begin{array}{l}\text { Normal } \\
\text { Anormal }\end{array}$ & $\begin{array}{r}22 \\
9\end{array}$ & $\begin{array}{l}70.97 \\
29.03 \\
\end{array}$ & $\begin{array}{l}5 \\
6 \\
\end{array}$ & $\begin{array}{l}45.45 \\
54.55 \\
\end{array}$ \\
\hline $\begin{array}{l}\text { Aceptación de } \\
\text { menstruación }\end{array}$ & $\begin{array}{l}\text { Si } \\
\text { No }\end{array}$ & $\begin{array}{l}17 \\
14\end{array}$ & $\begin{array}{l}54.84 \\
45.16\end{array}$ & $\begin{array}{l}8 \\
3\end{array}$ & $\begin{array}{l}72.73 \\
27.27\end{array}$ \\
\hline $\begin{array}{l}\text { Antecedentes } \\
\text { sicosomáticos }\end{array}$ & $\begin{array}{l}\text { Sí } \\
\text { No }\end{array}$ & $\begin{array}{r}23 \\
8\end{array}$ & $\begin{array}{l}74.19 \\
25.81\end{array}$ & $\begin{array}{l}9 \\
2\end{array}$ & $\begin{array}{l}81.82 \\
18.18 \\
\end{array}$ \\
\hline Vida sexual & $\begin{array}{l}\text { Satisfactoria } \\
\text { No satisfactoria }\end{array}$ & $\begin{array}{r}8 \\
23\end{array}$ & $\begin{array}{l}25.81 \\
74.19\end{array}$ & $\begin{array}{r}1 \\
10\end{array}$ & $\begin{array}{r}9.09 \\
90.91 \\
\end{array}$ \\
\hline $\begin{array}{l}\text { Relaciones } \\
\text { familiares }\end{array}$ & $\begin{array}{l}\text { Adecuadas } \\
\text { Inadecuadas }\end{array}$ & $\begin{array}{l}21 \\
10\end{array}$ & $\begin{array}{l}67.74 \\
32.26\end{array}$ & $\begin{array}{l}5 \\
6\end{array}$ & $\begin{array}{l}45.45 \\
54.55\end{array}$ \\
\hline Vida afectiva & $\begin{array}{l}\text { Estable } \\
\text { Inestable }\end{array}$ & $\begin{array}{l}11 \\
20\end{array}$ & $\begin{array}{l}35.48 \\
64.52\end{array}$ & $\begin{array}{l}5 \\
6\end{array}$ & $\begin{array}{l}45.45 \\
54.55\end{array}$ \\
\hline $\begin{array}{l}\text { Ventajas } \\
\text { del dolor }\end{array}$ & $\begin{array}{l}\text { Si } \\
\text { No }\end{array}$ & $\begin{array}{r}22 \\
9\end{array}$ & $\begin{array}{l}70.97 \\
29.03\end{array}$ & $\begin{array}{l}5 \\
6\end{array}$ & $\begin{array}{l}45.45 \\
54.55\end{array}$ \\
\hline Identificación & $\begin{array}{l}\text { Padre } \\
\text { Madre }\end{array}$ & $\begin{array}{l}10 \\
21\end{array}$ & $\begin{array}{l}32.26 \\
67.74\end{array}$ & $\begin{array}{l}5 \\
6\end{array}$ & $\begin{array}{l}45.45 \\
54.55\end{array}$ \\
\hline Actitud & $\begin{array}{l}\text { Equilibrada } \\
\text { Anormal }\end{array}$ & $\begin{array}{l}17 \\
14\end{array}$ & $\begin{array}{l}54.84 \\
45.16\end{array}$ & $\begin{array}{l}7 \\
4\end{array}$ & $\begin{array}{l}63.64 \\
36.36\end{array}$ \\
\hline
\end{tabular}

Muestra la sintesis de los resultados de la evaluación clínica sicológica de la entrevista y la aplicación del cues- tionario, en las diversas áreas investigadas, tanto para las pacientes con laparoscopias positivas como negativas. 
CUADRO 10

\section{DOLOR PELVICO (42 PTES.) \\ EVALUACION SICOLOGICA CONCLUSIONES DIAGNOSTICAS}

\begin{tabular}{lrcrr}
\hline & LAPAPOSO & \multicolumn{3}{c}{ LAPAROS: } \\
\hline Normales & + & 29.03 & 3 & 27.28 \\
Trastornos de personalidad & 9 & 41.94 & 4 & 36.36 \\
Siconeurótico & 13 & 29.03 & 4 & 36.36 \\
\hline
\end{tabular}

NOTA: Pacientes normales sensiblemente iguales. mayor incidencia de siconeurosis en pacientes laparoscopia negativa

Del análisis de este cuadro podemos resumir nuestras conclusiones diagnósticas asi:

Pacientes normales sensiblemente iguales.
Ligero predominio de pacientes con trastornos de personalidad en las laparoscopias positivas.

Mayor incidencia de siconeurosis en pacientes con laparoscopias negativas.

\section{Comentarios y conclusiones}

En la evaluación clínica médica de nuestro estudio, apoyados en la laparóscopia, definimos dos clases de pacientes que consultan por dolor pélvico. Estamos de acuerdo con otros autores (2) (3) para quienes el síntoma dolor se ha interpretado como de causa orgánica, pero al profundizar en el estudio de la paciente puede ser peligroso no considerar el sintoma como de causa sicosomática; así, pues, existen dos grupos de pacientes con dolor pélvico: de causa orgánica y de causa sicosomática, confirmados en nuestro estudio como laparoscopia positivas y negativas .

Este conocimiento, amplía el campo en el manejo de estas pacientes y conduce al éxito no siempre quirúrgico del tratamiento y conseguirá mejorar las condiciones de vida de las mujeres que consultan por dolor pélvico.

De acuerdo con Duncan y Taylor (7) creemos que la tendencia a localizar el dolor sicógeno en la pelvis, se debe al compromiso del área sexual en el campo sicógeno

El grupo de mujeres de nuestro estudio, de acuerdo con otros autores (:), se encuentran por encima de los 30 años y coincidimos en otros hallazgos como multiparidad, raza y alteraciones en sus relaciones interpersonales.

Llama la atención en nuestro estudio la presencia de varicocele pélvico y adherencias no consideradas por otros autores, como causas frecuentes de dolor pélvico

Deben tenerse en cuenta los antecedentes quirúrgicos y en este análisis hallamos relación con la presencia de adherencias.

\section{Pruebas MMPI}

El alto puntaje alcanzado por los sujetos del programa, tanto en el grupo positivo como en el grupo negativo respecto a la escala $F$, que mide la validez total de la prueba, nos indica que no hubo una clara comprensión de muchos 
de los items, debido a que la prueba no se encuentra estandarizada para América Latina.

No se encontraron diferencias significativas entre los dos grupos en el puntaje de las otras escalas. nótese sí. que el puntaje es promedio alto, indicativo de presencia de desórdenes mentales.

El alto porcentaje $70 \%$ encontrado en los dos grupos en las escalas Hy, Es, PA, D, tetrada sicótica, no nos permite asegurar un comportamiento de tipo sicótico en las pacientes, ya que durante el tiempo que se trabajó en ellas y su anamnesis no mostraron índice de ello.

Por lo anterior se concluye respecto a la prueba MMPI:

Debe ser estandarizada en nuestro pais para obtener porcentaje de validez real.

Es una prueba que aplicada en pacientes de nivel educacional bajo, por lo extensa, se hace fatigante aun cuando no existe límite de tiempo, más de dos horas para contestar una prueba, impide al individuo una actitud calmada. reflexiva y sincera, alterando con esto el resultado.

De las 42 pacientes sólo 31 se presentaron a la prueba. De las 11 pacientes que no la realizaron había 9 con causa orgánica y 2 sin causa orgánica. Nos preguntamos entonces si el rechazo a la prueba sea indicativo que estas pacientes tengan mayor compromiso sicológico que las que completaron su evaluación.

\section{Cuestionario y entrevista}

Las pacientes del programa establecieron buena interrelación durante las entrevistas y en la mayoria de los casos hablaron ampliamente de sus conflictos, algunas solicitaron a la sicóloga orientación en sus dificultades y otras fueron conscientes de la necesidad de sicoterapia.
Algunas pacientes se mostraron inhibidas en los items referentes a sexualidad.

Las pacientes que pudimos identificar como mujer con dolor de tipo sicosomático, según nuestro estudio, presentan un patrón de evaluación sicológico que corresponde a: socialización anormal, alta productividad, bajo autoimagen, marcada insatisfacción de su vida sexual, inadecuadas relaciones familiares, vida afectiva inestable, identificación con la madre, alta incidencia de trastornos sicosomáticos asociados y aceptación de su menstruación como hecho fisiológico a pesar de no encontrar ellas conscientemente ventajas a su dolor.

Lo anterior nos da una clara descripción de la sintomatología de trastornos de tipo siconeuróticos, su alta productividad puede traducirse como una tendencia compulsiva a través de la cual encontraron un ligero alivio a sus tensiones y conflictos.

Por otro lado, las pacientes en las cuales aducimos como orgánica la causa de dolor se caracterizan por socialización normal, productividad normal. autoimagen adecuada, aceptación de su menstruación, antecedentes sicosomáticos detectables, mediana satisfacción en su vida sexual, adecuadas relaciones familiares, pero con vida afectiva ines. table; le encuentran conscientemente ventajas al dolor y se identifican con su madre.

Lo anterior nos describe la sintomatología de los trastornos de personalidad, manifestados principalmente a través del área sexual, sicosomática y vida afectiva.

Comparando ambos grupos encontramos en el grupo de pacientes con diagnóstico laparoscopia positiva:

Mayor socialización, mejor autoimagen y mejores relaciones familiares.

En el grupo con diagnóstico laparoscopia negativa: 
Más alta productividad, mejor aceptación de su menstruación, mejor formación de antecedentes sicosomáticos. más acentuada insatisfacción sexual.

Llama así la atención que las mayores ventajas conscientes del dolor las encontramos en el grupo de pacientes con causa orgánica y una mejor identificación con la madre en las pacientes sin causa orgánica.

Encontramos a través de nuestro estudio resultados acordes con lo que Breuer (5), Noyes (16) y otros autores habían anotado en años anteriores, en lo que respecta al grupo de pacientes laparoscopia negativas, pero también podemos establecer que el grupo con causa orgánica detectable, para nosotros responsable del dolor, presentan sintomas de alteraciones como trastornos de la personalidad y en varios grados trastornos siconeuróticos, probablemente asociados a la presencia por largo tiempo, de un dolor de causa orgánica.

Con esto podemos establecer que si bien es cierto, las pacientes con trastornos siconeuróticos y de personalidad pueden desarrollar dolor pélvico como síntoma y producto de su alteración emocional, no exclusivo de este grupo como lo acabamos de demostrar, nos planteamos entonces el interrogante: ¿Las alteraciones sicógenas que acompañan el grupo de pacientes de causa orgánica, desempeñan un papel desen- cadenante del síntoma dolor o son el resultado del mismo dolor?

Comparando nuestros resultados con los obtenidos por M. Renaer (18). los encontramos sensiblemente iguales, en lo referente a los aspectos sicológicos del dolor pélvico crónico; en este trabajo los autores no encontraron diferencias significativas o alteraciones sicológicas del grupo laparoscopia positivas y negativas con el grupo control.

Dos aspectos queremos destacar en nuestra evaluación sicológica:

1. La marcada insatisfacción sexual encontrada en el grupo sin causa orgánica; como Richter citado por Luban (14) enuncia: "Es la protesta que la mujer formula con su cuerpo a la insatisfacción de su vida conyugal".

2. Las pacientes con causa orgánica si encuentran ventaja social y emocional a la presencia del dolor, cuando esperábamos que esta incidencia fuera mayor en las pacientes sin causa orgánica. Suponemos entonces que en estas últimas el trastorno siconeurótico que las caracteriza reprime la conciencia de sus ventajas.

\section{Concluimos y recomendamos}

Que en el estudio de pacientes que consultan por dolor pélvico se tengan en cuenta los siguientes patrones de evaluación, para hacer diferenciación etio lógica entre lo orgánico y sicógeno:
EDAD:

NIVEL SOCIO ECONOMICO:

PARIDAD:

EDUCACION:

ESTADO CIVIL:

RAZA:

CICLO MENSTRUAL:

LOCALIZACION:

RELACION CON EL CICLO:

TIPO DE DOLOR:

IRRADIACION:

PROBLEMAS SOCIALES Y PERSONALES
Por encima de 30 años

Bajo

Multiparidad

Básica o intermedia

Casadas

Blanca o mestiza

Normal

Generalmente sí

Fosas iliacas

Agudo

Espalda

Dificultades familiares y matrimoniales 
El problema debe ser manejado de acuerdo con las necesidades individuales de la paciente, utilizando los medios adecuados como la laparoscopia; conformar un equipo médico-sicólogo, interesado en el estudio, para poder brindar a la mujer la mejor solución al dolor pélvico crónico.

\section{Resumen y conclusiones}

El dolor pélvico crónico es un síntoma difícil de tratar ya que a menudo no se encuentra causa orgánica responsable. Con frecuencia el médico diagnostica enfermedad inflamatoria pélvica, suministrando series de tratamiento antibiótico y anti-inflamatorio, cada vez que el dolor recurre.

Muchas intervenciones quirúrgicas se han realizado, para aclarar la patología del dolor pélvico, sin resultados satisfactorios.

De otra parte, las pacientes en las cuales no se encontró patología orgánica atribuible como causa del dolor, fueron catalogadas dentro del marco de trastorno sicológico.

La disponibilidad de la laparoscopia permite, en el presente, distinguir causas orgánicas, responsables del dolor pélvico.

Se considera, para efectos del estudio la definición sobre dolor pélvico: "Síntoma dolor, manifestado por la mujer, relacionado o no con el ciclo menstrual, de tres o más meses de evolución, motivo de consulta ginecológica de por lo menos una vez, sin que se haya establecido diagnóstico etiológico que lo justifique".

Motivados por estas consideraciones, los autores se proponen realizar una evaluación racional del problema, contemplando los aspectos clínicos y sicológicos, de un grupo de pacientes. valiéndose de la laparoscopia, como un medio objetivo, para determinar patología pélvica.

Las pacientes fueron evaluadas en el área sicológica aplicando: a) Entrevista personal. b) Cuestionario diseñado específicamente para este estudio. c) Aplicación del "test" MMPI (Minnesota Multiphasic Personality Inventory).

En el área médica, fueron evaluadas la totalidad de las pacientes por:

a) Anamnesis. b) Examen ginecológico. c) Laparoscopia diagnóstica.

Se investigaron un total de cuarenta y dos (42) pacientes. Se encontró en el área médica que en el $26 \%$ de ellas no se detectó patología orgánica (laparoscopia negativas).

Los hallazgos en el $74 \%$ "laparoscopia positivas" fueron: Varicocele pélvico y adherencias pélvicas, correspondiendo a $2 / 3$ del total y en tres pacientes, ambas entidades fueron coincidentes.

El $1 / 3$ restante de pacientes (laparoscopia positivas), correspondieron: endometrosis pélvica, obstrucción tubaria, hidrosalpinx, miomatosis uterina, salpingitis y quiste de ovario.

El tratamiento previo de estas pacientes estaba lejos de ser el pertinente (antibióticos, anti-inflamatorios, hormonal) y la evaluación clínica, no aportó datos de ayuda diagnóstica efectiva.

En el área sicológica, los rasgos: histeria, esquizofrenia, paranoia, depresión y sicoastenia o hipomanía, aplicando el MMPI, fueron las características predominantes del $70 \%$ del grupo. De estos los rasgos esquizofrenia, paranoia, depresión e hipomanía, conformaron nuestra tétrada sicótica.

No se puede afirmar que ese $70 \%$ en realidad sean sicóticas, puesto que la entrevista, la situación de prueba y el cuestionario, no revelaron esta ten- 
dencia sicótica de grupo. podríamos sí hablar, de trastornos de tipo neurótico, que aparecen por el componente histérico o por el tiempo de exposición al dolor, por un largo período, con los consecuentes desajustes en la vida afectiva, sexual, social y productiva de estas mujeres.

Las pacientes "Iaparoscopia negatıvas" no mostraron diferencia significante con el resto del grupo, considerando el tamaño de la muestra.

Los autores concluyen, que para llegar al diagnóstico real del dolor pélvico crónico, debe tenerse en cuenta tanto el aspecto somático como el sicológico, puesto que ambos factores son constantes y no individualizables, para tener un mejor enfoque y un racional manejo del problema.

Recomiendan, que el problema debe ser manejado de acuerdo con las necesidades individuales de la paciente, utilizando los medios adecuados y conformar un equipo médico-sicólogo, interesado en el estudio, para poder brindar la mejor solución a la mujer que consulta por dolor pélvico crónico.

\section{PELVIC PAIN}

\section{Summary and conclusions:}

For the study purposes, the following definition is given for pelvic pain: "Pain symptom suffered by a woman, either related or not to menstruation, of three or more months of evolution, causing at least one gyneacological consultation where no ethiological diagnoses justifying it has been determined".

Cronic pelvic pain is very difficult to treat because usually no responsible organic cause can be found.

At present, by laparoscopy, it is possible to determine the organic causes of pelvic pain.
The patients were assessed in the psycological area by means of:

a) Personal Interview.

b) A questionnaire specifically desig. ned for this study.

c) MMPI Test (Minnesota Multiphasic Personality Inventory).

All the patients were evaluated in the medical field by:

a) Anamnesis.

b) Gynecological examination.

c) Diagnostic laparoscopy.

Fourty-two (42) patients were exa-
mined.

In the medical field it was found that in $26 \%$ of them no organic pathology was recorded (Negative Laparoscopy).

The findings of "Positive Laparoscopy" in $74 \%$ were: Pelvic Vericocele and pelvic adhesions, accounting for $2 / 3$ of the total, and in three patients both en. tities were coincider,tal.

The authors concluded that in order to obtain a real diagnosis for cronic pelvic pain, both the somatic and psychological aspects should be taken into consideration since both factors are constant and non individualized, thus having a better aproach and a rational handling of the problem.

They recommend to handle the problem according to the individual needs of the patient, using suitable means, and to form a medical-psychological team, interested in the study, in order to give the best solution to a women suffering from cronic pelvic pain.

\section{Referencias}

1 ANASTASI, ANNE: Test Psicológicos. Edit. Aguilar 1967. Capt. 18.

2 BEARD, R. W. BELSY, E. M. LIEBERMAN, B. A., WILKINSON, J. C. M.: Pelvic pain in women, M. J. Obstet. Gynecol. 128: 566 , 1977. 
3 BENSON, R. C.: Dolor pélvico sicógeno. Clinic. Obst. y Ginec. Edit. Interamericana. Marzo 1965. Pág. 161.

4 BERNAL, R.: Proyecto multidisciplinario e interinstitucional de bienestar social. Tesis Máster en Administración Industrial. Universidad del Valle, mimeógrafo, Mayo 1969

5 BREUER, JOSEF.: Contribuciones a los es. tudios sobre histeria. Edit. Siglo XXI. 1976. Págs. 133-145.

6 CROW, S., and CRISP., A. H.: A short cli. nical diagnostic selrating scale for psycho. neurotic patients: The Midlese Hospital Questionnaire, Br. J. Psychiatry 112: 917. 1963.

7 DUCAN, C. H., and TAYLOR, H. C.: A psychosomatic study of pelvic congestion, Am J. Obstet. Gynecol. 64: 1, 1952.

8 EYSENCK, H. J. and EYSENCK, S. B. G.: Manual of the Eysenck personality inven. tory, London, 1964, University of London Press.

9 FLOYD, L. RUCH.: Psicologia y Vida. Edit. Trillas 1971. Págs. 332-333.

10 FREUD: Inhibición sintoma y angustia. Edit Grigalbo 1970. Tres $\in$ nsayos sobre teoria sexual. Edit. Alianza. Editorial 1975.

11 FREUD: Escritos sobre histeria. Alianza editorial, 1975. Págs. 31, 34, 35, 133.

12 HATHAWAY, S. R.; MCKLINLEY, J. C : Manual MMPI. Edit. El Manuel Moderno S. A. 1976
13 KALK, cita por MARLOW. J.: Historia de la laparoscopia, sistema óptico y fibróptico e instrumentación. Clin. Obst. y Ginec., Edit. Interamericana. Junio, 1970.

14 LUBAN - PLOZZA LOCARNO: El enfermo psicosomático y el Médico práctico, Edit. Roche 1975, Capts. 1. 3, 12, 13

15 MANDY, T. E., WELNBERG, P., TUDOLPH, A.: Sychosexual conflicts: Their implications in functional pelvic disorders: Sothen Med. J. 48: 533, 1955.

16 NOYES, ARTHUR P.: Psisuiatria clínica moderna. Ed. La Prensa Médica Mexicana 1958, Capts. 5, 7, 23, 29

17 OSGOOD, C. E., SINCI, G. J., and TEMEN. BAUM, P. H.: The Measurement of Meaning. Chempaing, 111, 1957, University of Illinois Press.

18 RENAER. M., VERTOMMEN, H, NIJS. P., WAGEMANS, L.: Psychological aspects of chronic pelvic pain in women, Am J. Obst. y Ginecol. 134: 75, 1379 .

19 TAYLOR, H. C.: The problem of pelvic pain in Meigs, j. V . and somers, H. S. editors: Progress in Gynecology Vol. III. New York. 1957, Grune and Stratton. Inc., pág. 19.

20 TELINDE, R. W.: Culdoscopia. Aparato. Procedimiento. Indicaciones. Análisis, en ginecologia operatoria, Edit. Bernades, S. A., págs., 659-70, 1961 .

21 UDDENBERG, N.: Psychological aspects of sexual inadequacy in women, J. Psychosom. Res. 18: 33, 1974. 